Mots. Les langages du politique

\title{
Programmes et instructions officielles pour le français en Afrique francophone. Un rapport ambigu à la décolonisation
}

The official curriculum of French in French-speaking African countries. An ambiguous relation to decolonization

Programas y instrucciones oficiales para la enseñanza del francés en África francófona. Una relación ambigua con la descolonización

Michèle Verdelhan-Bourgade

CpenEdition

Journals

Édition électronique

URL : https://journals.openedition.org/mots/21760

DOI : $10.4000 /$ mots. 21760

ISSN : 1960-6001

Éditeur

ENS Éditions

Édition imprimée

Date de publication : 31 décembre 2014

Pagination : $27-42$

ISBN : 978-2-84788-544-6

ISSN : 0243-6450

\section{Référence électronique}

Michèle Verdelhan-Bourgade, "Programmes et instructions officielles pour le français en Afrique francophone. Un rapport ambigu à la décolonisation », Mots. Les langages du politique [En ligne], 106 | 2014, mis en ligne le 31 décembre 2016, consulté le 22 avril 2022. URL : http://

journals.openedition.org/mots/21760; DOI : https://doi.org/10.4000/mots.21760 


\section{Programmes et instructions officielles pour le français en Afrique francophone. Un rapport ambigu à la décolonisation}

Si l'on s'intéresse en tant que didacticien à la question de l'enseignement et de l'apprentissage du français en Afrique francophone, on est rapidement confronté à des questions connexes relevant du domaine institutionnel et politique. Par exemple, l'examen des manuels scolaires en usage ne peut être indépendant de celui des textes officiels qui régissent les programmes et contenus d'enseignement, et qui constituent la commande institutionnelle.

Les instructions officielles (désormais I0) et les programmes pour l'enseignement du français forment un ensemble de textes facilement identifiables. Pour l'Afrique francophone, ils ont peu fait l'objet d'études, les chercheurs s'étant plutôt intéressés aux lois fixant la politique linguistique (Halaoui, 1994, 1995), aux manuels scolaires ou aux pratiques d'enseignement. Nous nous interrogerons dans cet article sur cette commande officielle, échelon intermédiaire qui émane du politique et influe sur le terrain d'enseignement : la lecture des 10 nous surprenant par le mélange de préconisations parfois contradictoires, nous avons posé l'hypothèse qu'elles étaient le fruit d'influences variées voire rivales, que nous avons cherché à mettre au jour, et qu'elles sont par là même révélatrices de certaines contradictions qui parcourent les systèmes éducatifs africains aujourd'hui.

D'où quelques questions. Chaque pays de l'ex-empire colonial français dispose aujourd'hui de ses propres instructions officielles. Cinquante ans après les indépendances, quelles en sont les caractéristiques? Sont-elles spécifiques à chaque pays ou peut-on dégager des lignes générales? Quelles influences s'y font sentir? En particulier, se manifeste-t-il un rapport à l'ancienne puissance coloniale? Ou bien celle-ci a-t-elle été remplacée par d'autres influences? Comment, enfin, ces discours éducatifs traduisent-ils la complexité des tensions actuelles, intérieures aux pays ou même internationales? 


\section{Le corpus d'étude : nature et justifications}

\section{Les instructions officielles comme discours}

Nous considérerons les IO et les programmes d'enseignement du français comme des discours à traiter comme objets d'analyse pour plusieurs raisons :

- ils sont produits dans un contexte homogène, ancré dans l'institution éducative, quels que soient le pays et la date considérés;

- si on ne connaît pas précisément les auteurs de ces textes en personne, c'est parce qu'ils sont absorbés par l'institution qui donne son label; il est fréquent d'ailleurs qu'un discours introductif soit signé du ministre de l'Éducation en poste; l'anonymat des auteurs réels constitue de ce fait un obstacle à l'analyse, leur double statut, didactique et institutionnel, n'étant pas explicité (Moirand, 1988, p.128);

- leur destinataire est également un ensemble stable, englobant les acteurs de l'éducation dans le domaine considéré, à savoir les corps d'inspection, les chefs d'établissement, les enseignants, les parents d'élèves;

- comme on le verra plus loin, ils sont organisés de manière stable à une époque donnée;

- ils sont spécialisés dans un domaine correspondant à une matière ou discipline dont il s'agit de réguler l'enseignement.

Les textes officiels ont permis des études sur l'enseignement du français langue maternelle, par exemple Discours sur la lecture 1880-1980 (Chartier, Hébrard, 1989, p. 177-235), ou celui de l'histoire (Lécureur, 2012).

\section{Un discours mixte}

Leur origine et leur fonction donnent à ces discours un caractère mixte. On pourrait considérer que les 10 font partie des discours administratifs dont Gisèle Kahn a étudié les règles (2000). C'est de toute manière un discours institutionnel.

De plus, parce qu'il concerne le contenu et la manière d'enseigner une discipline, ce discours institutionnel s'appuie sur des courants didactiques qu'il promeut. En ce sens, c'est un discours didactique. Son articulation à la recherche contemporaine dans le domaine considéré est plus ou moins lâche, parfois affirmée, parfois pas, mais toujours implicite dans ce dernier cas.

Enfin, c'est un discours qui oriente, ou du moins qui donne des orientations supposées être suivies : c'est le sens de «instructions», qui s'imposent à tous et commandent les contenus, les modes de fonctionnement de la classe et les manuels scolaires. On a affaire ainsi à un discours de pouvoir (Boutan, 1996, Vargas, 1987). 


\section{Un discours de pouvoir au temps colonial}

Si l'on suit Denise Bouche (2000, p. 65-89), la puissance coloniale française est plutôt peu intervenue jusqu'à la fin de la seconde guerre mondiale dans l'organisation de l'enseignement dans les colonies, laissant surtout aux gouverneurs sur place le soin de le faire. Par contre, à partir de 1946 et de la création de l'Union française, ce sont les textes du ministère français de l'Éducation nationale qui vont régir les enseignements (Bouche, 2000, p. 84), et ce jusqu'aux indépendances, ou du moins jusqu'en 1957. Ce qui est à la fois logique et paradoxal : logique puisqu'il s'agit de renforcer les liens des pays colonisés avec la métropole en créant un grand ensemble qui se veut unifié avec des citoyens qui ont les mêmes droits, et paradoxal puisqu'en même temps des mesures budgétaires et administratives donnent une plus grande autonomie à ces pays.

En 1962, l'enseignement du français dans l'ex-AOF ou AEF était encore largement orienté par les instructions et programmes officiels français centralisés; ce n'est que progressivement que les pays indépendants créeront leurs propres textes.

\section{L'extension du corpus}

Nous avons choisi dans cette étude les instructions officielles pour l'enseignement du français au collège des huit pays suivants : Bénin, Burkina Faso, Cameroun, Gabon, Côte d'Ivoire (RCI), Congo (RDC), Mali, Sénégal.

Ce sont tous des pays de l'ex-champ colonial francophone, en Afrique de l'ouest ou Afrique centrale, qui se répartissent géographiquement entre Sahel, Côte Atlantique, zone équatoriale. L'ex-Empire colonial français, dit AOF et AEF, comportait en outre ce qui deviendra la Mauritanie, la Guinée, le Niger, le Tchad, le Togo, la République du Congo, la République centrafricaine. Le corpus couvre donc la moitié des anciennes colonies françaises en Afrique, à laquelle on a ajouté la république démocratique du Congo (RDC, ex-Zaïre) en raison de son appartenance à l'espace francophone et de sa population nombreuse qui en fait un des grands pays francophones d'Afrique.

Comme il ne s'agit pas d'une étude diachronique, les textes officiels sont ceux en vigueur en 2013. Ils peuvent avoir été rédigés les années précédentes (2006-2007 pour la RDC, 2010 pour le Burkina Faso et le Sénégal, 2011 pour le Gabon, par exemple). Ces variations seront considérées comme minimes dans la mesure où il n'y a pas eu de bouleversement méthodologique pendant la période 2006-2013 et où les références didactiques relèvent d'un même courant d'idées dominant, sur lequel on reviendra. On notera cependant que le rythme de parution des instructions officielles et programmes varie selon les pays : en Côte d'Ivoire ( $R C I)$, les IO de 2010 remplacent les précédents textes de 2006 en RDC (Congo Kinshasa), celles de 2007 succèdent à celles de 1970, 
ce qui peut s'expliquer entre autres par les événements de l'histoire politique de chaque pays.

Quant au niveau choisi, il correspond à la scolarisation des élèves de 11 à 15 ans, engagés dans un cycle d'enseignement qui porte des noms différents : enseignement secondaire (Bénin, Cameroun, RDC), cycle 1 de l'enseignement secondaire ( $\mathrm{RCl}$ ) ou de l'enseignement général (Burkina), cycle moyen (Sénégal), second degré (collèges) au Gabon, $6^{\mathrm{e}}, 7^{\mathrm{e}}, 8^{\mathrm{e}}$ et $9^{\mathrm{e}}$ années de l'enseignement fondamental au Mali. Dans la plupart des pays l'accès à ce niveau d'enseignement se fait par le filtre d'un examen d'entrée réputé difficile et discriminant. Les élèves de ces établissements sont donc engagés dans un processus de scolarisation sélectif, la deuxième étape de la sélection se situant à la fin de ce cycle, avant l'admission en lycée. C'est un cycle d'enseignement en expansion : après un temps où les efforts des gouvernements ont porté surtout sur l'enseignement primaire et l'alphabétisation, plusieurs pays lancent la réflexion sur les formations scolaires situées en amont (préscolaire) et en aval (collège). Le Gabon par exemple a préconisé en 2010 l'abandon de l'examen d'entrée en sixième afin d'ouvrir plus largement l'enseignement du second degré.

Les textes officiels à étudier émanent tous des ministères concernés, dont les appellations varient autour des termes enseignement (4 pays) ou éducation (4 pays), avec des extensions comme nationale (Sénégal, RCI), alphabétisation (Mali), instruction civique (Gabon), langues nationales (Mali), formation technique et professionnelle, reconversion et insertion des jeunes (Bénin). Les regroupements révèlent les préoccupations institutionnelles des pays concernés, parfois très techniques (primaire, secondaire, supérieur), parfois plus politiques : liaison avec la recherche, la formation professionnelle ou l'emploi, ou encore les langues nationales.

Ce corpus a été examiné selon quatre axes : sa présentation formelle, le statut du français tel qu'il est mentionné, les méthodologies préconisées et les références explicites et implicites qui les sous-tendent, les contenus d'enseignement prônés.

\section{Convergences et variantes}

Outre les constantes institutionnelles rappelées précédemment, les documents étudiés comportent un certain nombre de convergences, sur le plan du fond comme de la forme.

\section{La présentation et la structure des documents}

Tous les textes que nous avons consultés ont une présentation commune en deux parties : un discours linéaire suivi d'un tableau. Celui-ci peut avoir 
diverses fonctions : organiser les contenus selon des axes principaux, et/ou organiser le programme voire la progression de chaque année. Dans le premier cas, le tableau à double entrée comporte les compétences à acquérir dans l'année, puis les objectifs principaux et des contenus (Cameroun).

Dans le deuxième cas, l'organisation verticale est chronologique, avec ou pas l'indication des mois de l'année ( $R C l)$, des trimestres (Gabon) ou semestres, et horizontalement les différents domaines de l'enseignement : grammaire, vocabulaire, étude de textes, etc. Certains pays combinent les deux formules $(\mathrm{RCl})$. Un troisième cas utilise le tableau pour présenter un plan de séquence d'enseignement (Burkina Faso, $\mathrm{RCl}$ ). Pourquoi s'attarder sur cette forme de présentation? Provient-elle d'une tradition établie? L'auteur de ces lignes répondrait volontiers par la négative, se souvenant de ses étudiants africains en licence ou maîtrise, ou de professeurs en formation, qui éprouvaient beaucoup de difficultés à lire les tableaux grammaticaux à double entrée et expliquaient que cette forme de présentation ne leur était pas familière. S'agirait-il d'une imprégnation liée à la forme des programmes officiels français récents? Ce n'est pas le cas, ceux-ci (2008) étant présentés sous la forme de discours suivis et non de tableaux. On peut faire l'hypothèse que deux influences au moins jouent un rôle : celle de la présentation des contenus des manuels et méthodes de français langue étrangère ou de français langue seconde qui, depuis plus de trente ans pour les premières et vingt ans pour les secondes, organisent en tableaux la matière à enseigner; et celle de la méthodologie qui sert principalement de référence à ces textes officiels, et qui émane principalement du Canada et de la Belgique.

\section{La méthodologie de référence}

La plupart de ces textes font explicitement référence à une méthodologie connue : l'approche par compétences (APC). C'est le cas de la Côte d'Ivoire (p.3) : «une entrée dans les apprentissages par les situations en vue de développer les compétences chez l'apprenant en lui offrant la possibilité de construire le sens de ce qu'il apprend ». Le mot de la ministre de l'Éducation nationale cite nommément Philippe Jonnaert, «Professeur titulaire de la Chaire Unesco en Développement Curriculaire de l'Université du Québec à Montréal qui nous a accompagnés dans le recadrage de nos programmes éducatifs ». Le Sénégal revendique une «entrée par les compétences» (p. 2), le Cameroun s’inscrit aussi dans cette ligne. Le texte du Burkina Faso ne mentionne pas l'approche par compétences et utilise plutôt dans les tableaux le terme objectifs, mais s'appuie cependant sur cette approche. Le texte de la RDC affiche «une pédagogie par objectifs ou par compétences », apparemment considérées comme équivalentes, mais fait également référence à des méthodologies antérieures, communicatives et fonctionnelles. Seul le programme du Gabon semble éloigné d'une telle orientation. 
Cette référence constante à une méthodologie importée du Canada, qui se traduit dans la présentation formelle des 10 , recouvre cependant des disparités importantes dans son appropriation par les textes officiels, sur lesquelles nous reviendrons plus loin (voir «L’influence américano-canadienne»).

\section{La place du français}

La plupart des textes font état, dans un passage préalable (introduction, principes généraux, fondements), de la place particulière du français dans le pays considéré, langue officielle, seconde, en contexte national et international ${ }^{1}$. Nous citons en exemple les extraits suivants qui concernent la situation spécifique du français au Sénégal et en Côte d’Ivoire.

$10 \mathrm{RCl}, 6^{\mathrm{e}}-5^{\mathrm{e}}$ (p. 6)

Du fait de son statut de langue officielle en Côte d'Ivoire, le français est à la fois outil de communication et discipline d'enseignement parce que faisant lui-même l'objet d'apprentissage en vue de sa maîtrise pour une insertion harmonieuse du citoyen ivoirien dans sa société.

IO Sénégal (p.4)

1 - Statut du français

Langue officielle du Sénégal, le français joue un rôle fondamental dans notre système éducatif où il est à la fois médium et matière d'enseignement. En effet, la maîtrise du français s'avère indispensable pourl'acquisition des connaissances dans les autres disciplines, la poursuite des études, la communication sociale ou internationale. En outre, le français cohabite avec les langues nationales, dont certaines sont codifiées et enseignées dans le secteur non formel de l'alphabétisation fonctionnelle ou sont en cours d'expérimentation dans le secteur formel. L'évolution du français dans ce contexte de plurilinguisme et de nécessités lui confère donc un statut particulier, qui implique de ce fait, entre autres facteurs, une didactique appropriée.

Les Fondements (p.5) des IO de la RDC affirment qu'il s'agit d'une pédagogie «visant à la maîtrise d'une langue seconde», dont les objectifs seront de former le citoyen.

Le Burkina Faso ne fait pas mention de la place du français, le document du Gabon non plus, du moins dans la version que nous avons examinée, qui ne comporte que la progression annuelle.

\section{Convergences et différenciations de contenus}

Dans le détail des programmes se retrouvent de nombreux points communs. En grammaire ou conjugaison, par exemple, le programme est quasiment iden-

1. Les notions de français langue seconde ou de français langue de scolarisation ne peuvent être développées ici. On se référera à Cuq (1995) et Verdelhan-Bourgade (2002). 
tique d'un pays à l'autre (phrase simple, complexe, groupe nominal, etc.), et témoigne d'une même référence à une grammaire aux classifications «traditionnelles» (sujet, complément d'objet...) sur fond d'approche structurale (classes de mots, déterminants) ou énonciative. L'étude de textes et la production écrite renvoient à la classification en types de textes (narratif, argumentatif, descriptif...) et à la distinction entre explication de textes et lecture suivie. Un autre point commun concerne le peu de place accordé à la pédagogie de l'oral : si certains pays ont en effet une rubrique concernant l'expression orale, pratiquement aucun ne s'intéresse à la compréhension de l'oral.

Cet ensemble de convergences s'explique de manière tout à fait pragmatique d'abord par des constantes de terrain. Si la place du français est variable dans tous ces pays selon l'existence ou non d'autres langues officielles ou le rapport aux langues nationales (plus ou moins nombreuses, entre 250 et 270 au Cameroun, 13 langues nationales et une quinzaine dites régionales au Sénégal), le français est toujours langue officielle, seconde et de scolarisation (cf. note 1), du fait qu'il est la langue de l'ancien colonisateur. Les pays ont en effet, au moment de leur indépendance, fait le choix du français comme langue officielle, langue de l'administration et langue de l'enseignement, et œuvrent depuis en interne pour allier ce choix avec celui de la reconnaissance de leurs langues locales (Gabon, États généraux, 2010), qui se réalise surtout au primaire, et différemment selon les pays (LASCOLAF, Maurer, 2010).

De ce fait, les problèmes d'apprentissage sont à peu près identiques : ils concernent une même langue, le français, qui n'est pas dans la plupart des cas langue maternelle de l'élève, bien qu'étant langue officielle et donc présente dans l'environnement à des degrés divers ${ }^{2}$. On les retrouve d'ailleurs dans d'autres situations : le système des temps et des modes, l'usage des pronoms, l'organisation de la phrase sont cause de difficultés d'apprentissage du français partout dans le monde, quelle que soit la langue première de l'élève. De ce point de vue, l'apport de la comparaison avec la langue de départ n'est pas forcément capital : son principal intérêt est d'éveiller l'esprit à la relativité des langues, d'autant qu'à ce niveau on a affaire à des adolescents. On touche là d'ailleurs à une autre constante, celle de l'âge des élèves, de 11 à 15 ans, dont on peut supposer que les capacités cognitives sont identiques quel que soit le pays considéré, et qui sont engagés dans une scolarité souvent coincée entre deux sélections : celle de l'entrée en sixième, qu'ils ont franchie avec succès, et celle de la fin du collège (on le dira ainsi pour simplifier), qui donnera ou pas accès au lycée.

Ces contraintes communes expliquent donc un certain nombre de convergences. Mais chaque pays a construit des textes officiels spécifiques en

2. La situation sociolinguistique est certes variable selon les pays, les zones rurales ou urbaines, les régions. Les textes officiels pour l'enseignement du français adoptent cependant une vision générale de la place du français dans le pays. 
fonction de ses propres choix. L'identité patriotique est parfois nettement affirmée, comme dans les programmes de RDC dont les fondements ou «principes qui guident l'action pédagogique» découlent notamment du «profil de l'homme congolais à former» (p. 5), ce qui conduit à «éveiller à la conscience nationale » ou à « développer les valeurs républicaines». La thématique choisie chaque année reflète des préoccupations nationales : en classe de sixième, par exemple, on parlera des droits de l'enfant au Burkina Faso, de l'orientation et de l'entrepreneuriat ou du civisme fiscal en $\mathrm{RCl}$, de l'éducation à l'image au Sénégal ou des médias et de la communication au Cameroun. Qu'on y retrouve des thématiques communes comme la prévention des maladies (notamment le sida) ou la vie quotidienne relève là encore des constantes de terrain.

Outre les thématiques, les textes officiels se différencient par des éléments formels : présence ou pas de guides pédagogiques pour l'enseignant (exemples de démarches, de leçons) et leur degré de détail, présentation d'une progression sur l'année ou seulement indication de lignes générales.

On voit ainsi apparaître à travers les textes le travail d'équipes nationales qui s'efforcent d'allier les choix nationaux avec des références didactiques générales. D’où proviennent ces références et comment s'ajustent-elles aux pays?

\section{Le jeu des influences}

Malgré son caractère vague, nous utiliserons volontairement le terme d'influence, qui couvre des références éducatives transmises par des voies diverses : travaux scientifiques, conférences, séminaires de formation d'enseignants, aide au développement...

Deux grands champs d'influence semblent peser sur les textes officiels concernant l'enseignement du français dans les pays considérés : l'influence française (ou belge en RDC) et l'influence anglo-américaine.

\section{L'influence française}

La première est logiquement attendue du fait du passé colonial. Mais les IO africaines ne sont pas le calque des 10 françaises; on l'a par exemple vu dans leur présentation. L'influence française se fait plutôt sentir dans l'importance accordée, d'une part, à la typologie des textes, d'autre part aux études de la langue (surtout grammaire, conjugaison, orthographe) dans la plupart des programmes. On la retrouvera également dans le cloisonnement souvent affiché (grâce à la disposition en colonnes) entre ces différents domaines, alors même que le discours introductif insiste non sur des savoirs disciplinaires, mais sur des compétences à acquérir, lesquelles sont transversales. 
L'étude des textes semble bien relever d'une tradition scolaire française, avec son découpage en explication de textes (Burkina Faso), ou lecture méthodique et exploitation de textes, ou lecture analytique d'un côté, et lecture suivie ou lecture d'œuvre intégrale (RCI, Burkina Faso, Cameroun, Sénégal) de l'autre. De plus, la typologie proposée au tournant des années quatre-vingt/quatrevingt-dix par Jean-Michel Adam (1992) ou André Petitjean (1989) demeure très présente en Côte d'Ivoire, au Cameroun, au Sénégal. La division en textes descriptif, narratif, argumentatif, dialogal, reste en effet un quasi-dogme qui structure la vision de l'enseignement. On retrouve également certaines constantes des programmes français, comme l'étude des paramètres du récit selon Propp (situation initiale, finale, éléments déclencheurs etc.).

Les études de la langue occupent certes encore une position forte dans les programmes français du collège (2008). Mais si l'on compare les contenus proposés pour la classe de sixième en France et dans certains pays, on s'aperçoit qu'ils sont beaucoup plus copieux en Afrique (un seul présente un programme moins dense). Dans tous les programmes étudiés, l'accent est mis sur l'orthographe et la conjugaison et l'on peut y voir dès les classes de sixième et cinquième (première et deuxième années du collège) des points aussi difficiles que l'accord des adjectifs de couleur ou le pluriel des noms et adjectifs composés.

Cet accent mis sur les études de la langue nous paraît toutefois relever d'un héritage double : celui de l'enseignement linguistique formel, tel qu'il était pratiqué en France et dans son champ jusque dans les années soixante-dix, et celui d'une pédagogie centrée sur la transmission des savoirs, dans laquelle la connaissance est le pivot de la formation des individus. C'est bien à cette pédagogie qu'étaient attachées les élites africaines lorsqu'elles récusaient en 1937, par la voix de Senghor, les écoles rurales développant des savoir faire manuels au détriment des savoirs théoriques (Bouche, 2000, p.75). Le savoir, et notamment celui concernant les formes de la langue ou les textes littéraires, apparaissait comme le seul moyen de réussite sociale dans une société régie par la colonisation. Était-ce dû à l'influence du modèle culturel du colonisateur qu'il s'agissait de s'approprier pour mieux le combattre? Ou aussi à celle d'un modèle transmissif du savoir bien ancré dans la société africaine? Cette forte prééminence actuelle de l'apprentissage linguistique apparaît en tout cas en continuité avec ce que Pierre Dumont décrivait dès 1986 (p.38) : «cette conception élitiste et culturelle de la francophonie [...] indissociable d'un attachement très solide aux formes classiques, ou pseudo-classiques, de la langue française. »

Le choix des textes proposés aux élèves représente un degré supérieur d'autonomisation par rapport à la référence coloniale. Certes, on y trouve des auteurs français tels que Molière, Corneille, Racine, Hugo... attribués aux mêmes classes qu'en France (Le Cid en quatrième, Cameroun). Mais la part principale est accordée aux auteurs africains, d'abord ceux du pays considéré, puis ceux d'Afrique de façon plus large. On pourra trouver ensuite, dans une 
moindre mesure, ceux de la francophonie (Aimé Césaire et Guy Tirolien sont les deux auteurs francophones non africains cités par les 10 pour les classes de sixième et cinquième au Sénégal). Ces choix d'auteurs africains traduisent la volonté d'enracinement « dans les valeurs de culture pour mieux aller à la rencontre de l'Autre », pour reprendre la formule du texte sénégalais dans l'exposé des motifs. Ce n'est pas une nouveauté en soi, les manuels anciens comportaient déjà des textes d'auteurs africains, mais c'est la proportion qui change.

\section{L’influence américano-canadienne}

Le deuxième facteur d'influence appartient à un champ plus large, à la fois au plan géographique et notionnel. C'est celui qui pèse sur la didactique des langues vivantes depuis bon nombre d'années, et qui s'est traduit au plan méthodologique successivement par la "pédagogie par objectifs» puis par l'«approche par compétences» (APC).

\section{Les origines et supports de ces courants}

Les notions de pédagogie par objectifs et d'APC sont nées aux USA. La première, dans les années trente avec Ralph Tyler puis développée par Benjamin Bloom, visait à adapter les comportements professionnels aux besoins du travail et de la société. La seconde est apparue dans les années soixante (Competency-based education) comme prolongement et dépassement de la précédente (Ghosn, 2012, p.118), dans un monde économique où il s'agissait de mettre en avant l'adaptabilité à la tâche plutôt que le diplôme ou les connaissances. Toutes deux se sont rapidement étendues à d'autres pays, d'abord au Canada, puis en Europe, où d'importants relais francophones de la pédagogie par objectifs ont été Philippe Meirieu (France) ou Jean-Marie de Ketele (Belgique), pédagogie qui a eu une forte influence de 1980 à 2000 environ.

Québec et Belgique ont été les premiers pays à bâtir des programmes d'éducation sur la base des compétences de l'élève et ont fortement contribué à diffuser cette approche dans d'autres pays, notamment par l'intermédiaire d'experts comme Philippe Jonnaert (Québec) ou Xavier Roegiers (Belgique), très présents dans les pays africains, ou Mohamed Miled (France, Tunisie). Depuis le début des années 2000 et l'élaboration du Cadre européen commun de référence pour les langues, l'APC a fait ainsi l'objet de multiples conférences, séminaires et formations dans les pays africains, auprès des cadres de l'Éducation, en prônant notamment dans la dernière période le remplacement des programmes d'enseignement par des curriculums basés sur des compétences: «l'élaboration du curriculum consiste principalement à définir des finalités éducatives, à établir les besoins des apprenants, à déterminer des objectifs, des contenus, des démarches, des moyens d'enseignement et des formes d'évaluation ». (Martinez, 2002, p. 10). La notion de curriculum en éducation 
provient des pays anglophones et ne doit pas être confondue avec celle de programme, familière aux pays d'influence francophone : «le programme est le résultat de décisions relatives au curriculum » (ibid., p. 13).

Bruno Maurer (2011) pose une analyse idéologique de ces orientations méthodologiques, liées selon lui à l'expansion de la pensée libérale en économie à partir de l'Amérique du Nord dans la deuxième moitié du $20^{e}$ siècle. À titre d'exemple, constatons simplement dans les IO l'abus du verbe "gérer», abondamment utilisé dans le monde économique libéral, pour définir parfois des compétences bien simples : "gérer des situations dans lesquelles l'élève doit produire des énoncés oraux», "gérer des situations dans lesquelles l'élève doit construire le sens de textes divers» $(\mathrm{RCl})$. Ce qui peut se traduire par : faire parler et lire pour apprendre à parler et à lire.

\section{L'APC et les textes officiels : les difficultés observées}

Un rapport de 2010 (Cros et al.), commandité par plusieurs institutions', fait un état des lieux des réformes curriculaires dans cinq pays qui ont introduit l'approche par compétences : Cameroun, Gabon, Mali, Sénégal, Tunisie. Le constat, sévère, porte tant sur les modalités d'introduction des réformes que celles de leur réalisation, sans toutefois remettre en cause, ou seulement timidement, la pertinence du choix initial.

Le rapport 2010 présente l'APC comme solution aux maux de l'école africaine héritée de la colonisation : "elle semble le remède miracle susceptible de mettre fin aux maux des systèmes scolaires africains et de résoudre dans un court laps de temps ce qui est vécu comme un décalage par rapport aux pays de l'OCDE» (p.70). La croyance toutefois ne fait pas tout et la question de l'adaptation au terrain, déjà signalée par Verdelhan-Bourgade en 2007 (p. 110-122), se trouve ici fortement posée (Cros, p. 72) :

La question du contexte et des conditions réelles de fonctionnement des systèmes éducatifs concernés ne semble pas posée par rapport à la pertinence de la réforme. On peut dès lors s'interroger sur la qualité de l'analyse préalable de faisabilité de ces réformes : par rapport à quelle analyse des conditions réelles de fonctionnement des systèmes éducatifs locaux a été défini le bien-fondé du choix de l'APC comme réforme curriculaire? La question de la pertinence de la réforme APC ne semble pas avoir été clairement posée. Tout se passe comme si le choix de l'APC relevait plus d'une conviction intellectuelle et théorique que de l'analyse de la pertinence de la réforme par rapport à un contexte précis et des objectifs définis.

Certaines difficultés relèvent bien de la non-congruence entre les préconisations de l'APC et le terrain. C'est peut-être ce qui explique les degrés divers d'appropriation de l'APC par les IO, où l'approche par compétences se trouve parfois fort malmenée. En RDC la pédagogie par objectifs reste dominante,

3. Groupe de la Banque africaine de développement, AFD, OIF, CIEP. 
même si les IO affichent des termes issus de l'APC. Parfois la divergence est plus forte. Alors que dans l'APC les savoirs linguistiques sont à mettre au service des compétences de production orale ou écrite et ne font pas l'objet d'une visée d'apprentissage en soi, des programmes présentent pour l'essentiel des contenus linguistiques énormes, y compris en expression écrite ("ponctuation, les registres de langue, enrichir la phrase avec des expansions»). Parfois, le souci de fusionner l'apprentissage du savoir linguistique avec une approche qui met la situation de vie comme point de départ conduit à des contradictions : «Exemple de situation. Dans une situation de communication précise, l'élève doit utiliser la pronominalisation » $\left(\mathrm{RCl}, 5^{\mathrm{e}}, \mathrm{p} .51\right)$. Or la pronominalisation (comme bien d'autres faits grammaticaux) n'est pas liée à une situation de communication précise.

Ce poids du contenu linguistique rend inopérants les efforts de présentation des instructions en termes de compétences. Notamment parce que les enseignants vont devoir passer le plus clair de leur temps à réaliser l'énorme programme linguistique imposé et non à développer l'expression écrite ou orale.

De grandes différences se manifestent ainsi selon les pays entre le choix théorique de l'APC et sa traduction dans les programmes. C'est peut-être là que réside le plus grand mystère des 10 et programmes pour l'enseignement du français en Afrique : dans le contraste entre la théorie affichée, les gros efforts financiers et humains faits pour introduire la méthodologie APC dans les pays, et ce qui en ressort dans les textes officiels.

\section{Un rapport ambigu à la colonisation-décolonisation}

Revenons tout d'abord sur nos deux principaux constats concernant les textes officiels sur l'éducation : le statut du français, les incohérences des préconisations.

\section{Le statut peu clair du français}

Quel est le statut exact du français visé par ces textes officiels? Comme on l'a dit plus haut (voir "La place du français»), le français est affirmé comme langue seconde, langue officielle mais non maternelle pour la plupart des élèves. On pourrait penser que les instructions et programmes prennent en compte cette réalité. Or ce ne semble pas être le cas. Les savoirs linguistiques, langue et texte, demandés sont parfois plus complexes à un niveau donné que ceux qui sont exigés en France. Des besoins spécifiques à l'apprenant de langue seconde, comme la compréhension de l'oral, le vocabulaire thématique ou le travail sur la prononciation, ne sont pas ou sont peu pris en compte. Les traces de la situation africaine se trouvent dans le choix des textes à étudier, qui mêle 
auteurs africains et français, et des thèmes imposés, comme les séances obligatoires sur le sida, dès la classe de $6^{\mathrm{e}}$. La méthodologie d'apprentissage, elle, ne s'intéresse pas à la position réelle de l'apprenant de langue non maternelle. En ce sens, on peut avancer que la conception de l'enseignement du français reste marquée par l'héritage de la période coloniale, pendant laquelle il s'adressait soit à des locuteurs de français langue maternelle, soit à une future élite de la nation.

\section{Pourquoi un tel déséquilibre entre moyens investis et résultats?}

Les difficultés importantes relevées dans les 10 concernant la préconisation de l'APC aboutissent à des contradictions internes qui rendent difficile la mise en œuvre de cette démarche. Et pourtant des moyens humains et financiers très importants ont été injectés dans la formation à cette méthodologie. L'inadéquation de la démarche au terrain africain peut être une première réponse, comme on l'a signalé. Est-ce la seule? En utilisant une formule que Ouane et Glanz appliquent dans une autre problématique (2011, p. 269), pourquoi «poursuivre les investissements dans des programmes voués à l'échec »? On peut avancer plusieurs hypothèses.

\section{Des tensions internes}

L'ambiguïté dans le traitement du français et le degré d'appropriation divers des courants pédagogiques importés ne sont-ils pas signes d'une tension multipolaire qui se retrouverait dans les équipes rédactionnelles des 10 ? Par exemple :

- entre les défenseurs d'une pédagogie des savoirs (les «anciens», qui imposent des contenus très lourds en orthographe, grammaire et conjugaison) et ceux d'une pédagogie par compétences qui limite les savoirs (les «modernes»)?

- plus subtilement, si la «modernité» considérée comme attractive se trouve du côté de l'Occident (Dufour, 2007), serait-ce une tension entre cette influence moderniste et la volonté d'affirmer l'autonomie intellectuelle et pédagogique du pays? Tension que Hélé Béji (2008, p.106) analyse comme particulière au "décolonisé »: "Au désir de progrès se mêle quelque chose qui n'est plus dans la continuité de la civilisation [...], qui la fait sortir de la modernité proprement dite et qui, soudain, lui fait dépasser l'histoire européenne par une inspiration singulière : le refus viscéral de sacrifier sa tradition »;

- entre des «idéalistes» imbus de théories récentes, considérant l'APC comme le nec plus ultra de la pédagogie, et des «réalistes » conscients des difficultés sur le terrain et des habitudes pédagogiques?

- entre la culture pédagogique à la française et l'influence nord-américaine? Cette dernière pouvant de plus apparaître comme «libératrice» par rapport à l'ancienne puissance coloniale? 


\section{Une pénétration idéologique}

Pourtant, à partir du moment où un modèle éducatif est importé à coup de missions d'experts étrangers et de subventions, sans réelle étude préalable d'adéquation avec la réalité du terrain, n'y a-t-il pas là comme une forme de néocolonisation? On peut récuser l'expression, mais on ne peut pas nier la pénétration idéologique, l'empreinte d'une pensée venue d'ailleurs. Peut-on parler de colonialisme éducatif mondialisé, comme on parle de mondialisation en matière économique? Certes, les gouvernements ont donné leur accord; certes, il n'y a aucune raison pour que les pays africains restent à l'écart des évolutions pédagogiques mondiales; certes, l'amélioration de la scolarisation reste un objectif capital de développement. Certes enfin, les réticences observées à l'encontre d'innovations pédagogiques majeures ne sont pas forcément justifiées. On en avait déjà observé, dans les années quatre-vingt-dix, à propos de la pédagogie du langage et de la communication, destinée à motiver les jeunes enfants et à faciliter l'apprentissage du français. Il n'empêche que des solutions conformes aux conditions d'enseignement au Canada peuvent difficilement être importées telles quelles dans les pays d'Afrique, sinon cela revient à refaire en partie les mêmes erreurs que celles imputées à la colonisation.

\section{Le savoir, «bien commun planétaire»}

La question rejoint la critique apportée à l'aide au développement, auquel il est fait le reproche d'être l'héritier de la colonisation. Sans entrer dans ce débat complexe, nous noterons que le savoir, comme l'environnement ou la santé. fait partie de ce qu'on appelle depuis 1999 les «biens communs planétaires », dont l'accroissement et la répartition relèvent d'une «coopération internationale qui englobe tous les pays, et pas seulement les rapports entre le Nord et le Sud». (Severino, 2001, p. 36). Il n'empêche que la coopération internationale n'est pas égalitaire, en raison de la puissance économique et financière des pays. C'est ainsi qu'on a vu se succéder par exemple au Mali, depuis 1990, dans le secteur de l'éducation, parallèlement au déclin financier de l'aide française, l'augmentation de l'appui canadien, puis américain.

Dans cette logique, la rédaction laborieuse des textes officiels sur l'éducation et son résultat parfois ahurissant au plan didactique pourraient être liés à une vision de l'éducation africaine comme devant se rapprocher d'un modèle occidental. Et ce rapprochement se fait dans une tension entre tradition pédagogique locale, héritage de la colonisation française ou belge, et néocolonialisme américain. L’Afrique est toujours un enjeu de pouvoir entre grandes puissances. On n'est pas là seulement dans un conflit culturel entre deux types de pédagogie, mais aussi dans une lutte politique et économique.

Les textes officiels africains francophones sur l'éducation portent la marque des efforts fournis par chaque pays pour se doter d'un instrument propre de 
développement, permettant de s'émanciper d'un passé colonial. Mais le souci de s'aligner sur le modèle éducatif et didactique dominant en Occident conduit à un décalage et à un paradoxe. Décalage parce que le modèle choisi entre en contradiction avec le terrain : tradition pédagogique de transmission des savoirs, effectifs des classes, manque de matériel, formation des enseignants... Paradoxe parce que l'effort de développement éducatif autonome, qui aboutit ainsi au placage d'un modèle extérieur à une situation nationale, revient finalement, au nom de la modernité et du progrès pédagogique, à un mécanisme proche de celui reproché à juste titre aux anciennes puissances coloniales. Parce que les instructions officielles sur l'éducation sont des discours de pouvoir interne, elles sont entrées comme enjeu dans les rapports de force internationaux.

\section{Références}

AdAM Jean-Michel, 1992, Les textes. Types et prototypes, Paris, Nathan.

BÉıl Hélé, 2008, Nous, décolonisés, Paris, Arléa.

Bouche Denise, 2000, «Dans quelle mesure Paris a-t-il voulu diriger l'enseignement colonial? ", L'enseignement et la diffusion du français dans l'Empire colonial français, 1815-1962, G. Vigner éd., Documents pour l'histoire du français langue étrangère ou seconde, $\mathrm{n}^{\circ} 25$, p. 65-89, Paris, SIHFLES.

BoutAn Pierre, 1996, La langue des messieurs, Paris, A. Colin.

Chartier Anne-Marie, HÉBrard Jean, 1989, Discours sur la lecture, Paris, Centre Pompidou, BPI.

CROS Françoise et al., 2010, Étude sur les réformes curriculaires par l'approche par compétences en Afrique, CIEP, MAE, Groupe de la Banque africaine de développement, OIF, AFD.

CUQ Jean-Pierre, 1991, Le français langue seconde, Paris, Hachette.

Dufour Françoise, 2007, Des rhétoriques coloniales à celles du développement. Archéologie discursive d'une dominance, thèse de doctorat, Montpellier 3.

Dumont Pierre, 1986, L'Afrique noire peut-elle encore parler français? Paris, L'Harmattan. Gabon, mars 2003, États généraux de l'éducation, de la recherche et de l'adéquation à l'emploi, Libreville, Actes, http://www.gouvernement.ga/site/pdf/actesversiondef.pdf (consulté le 3 juillet 2014).

GHosn Racha Jaber, 2012, De la conceptualisation de la compétence à sa modélisation didactique, thèse pour le doctorat en sciences du langage sous la direction de M. Verdelhan, Montpellier 3.

Halaou Nazam éd., 1994, «La langue, la loi et la francophonie», Universités, nº13 (2), p. 21-44.

- 1995, Lois et règlements linguistiques des États francophones, Paris et Talence, ACCT - DGEF - EIB.

KAHN Gisèle, 2000, "La langue de l'administration », Histoire de la langue française 1945-2000, B. Cerquiglini, G. Antoine éd., Paris, CNRS, p. 294-311.

KETELE Jean-Marie (DE), 2000, «Approche socio-historique des compétences dans l'en- 
seignement», C. Bosman, F.-M. Gérard, X. Roegiers éd., Quel avenir pour les compétences? Bruxelles, De Boeck Université, p. 83-92.

JONNAERT Philippe, 2009, Élaborer et évaluer des manuels scolaires. Document de travail, N’Djamena, Tchad, http://www.cudc.uqam.ca/articles/20.\%20Manuels\%20 scolaires.pdf (consulté le 3 juillet 2014).

LÉCUREUR Bertrand, 2012, Enseigner le nazisme et la Shoah. Une étude comparée des manuels scolaires en Europe, Braunschveig, Georg Eckert Institut.

Martinez Pierre éd., 2002, Le français langue seconde. Apprentissage et curriculum, Paris, Maisonneuve et Larose.

MAURER Bruno, 2010, Les langues de scolarisation en Afrique francophone, Enjeux et repères pour l'action, Rapport général du projet LASCOLAF. AUF, OIF, AFD, MAEE, http://www.elan-afrique.net/wp-content/uploads/2011/o6/RapportDeSyntheseLight.pdf (consulté le 3 juillet 2014).

- 2011, Enseignement des langues et construction européenne. Le plurilinguisme, nouvelle idéologie dominante, Paris, Archives contemporaines.

Mı́CHÉA Jean-Claude, 1999, L'enseignement de l'ignorance et ses conditions modernes, Castelnau-le-Lez, Climats.

MILED Mohamed, 2004, "Les apports de l'approche pédagogique par compétences à la didactique du FLS», Marges linguistiques, juillet.

MoIRAND Sophie, 1988, Une histoire de discours, Paris, Hachette.

OuAne Adama, Glanz Christine éd., 2011, Optimiser l'apprentissage, l'éducation et l'édition en Afrique : le facteur langue. Étude bilan sur la théorie et la pratique de l'enseignement en langue maternelle et l'éducation bilingue en Afrique subsaharienne, Hambourg et Tunis, Unesco (UIL) et ADEA.

Petitjean André,1989, "Les typologies textuelles», Pratiques, n62, p. 86-125.

Roegiers Xavier, 2002, Élaborer un curriculum en termes de compétences : enjeux et démarches, Paris, Agence internationale de la francophonie.

SEVERINo Jean-Michel, 2001, "Refonder l'aide au développement au XXIe siècle», Critique internationale, 1, no 10, p.75-99, www.cairn.info/revue-critique-internationale-2001-1page75.htm (consulté le 3 juillet 2014).

VARGAS Claude, 1987, Langage et norme(s) à l'école primaire. Analyse sociolinguistique des textes officiels de la Révolution à nos jours, Aix-en-Provence, Université de Provence.

VERDELHAN-BOURGADE Michèle, 2002, Le français de scolarisation. Vers une didactique réaliste? Paris, PUF.

- 2007, «Réflexion sur l'approche par compétences et sa pertinence pour l'enseignement du français langue seconde», Le français langue seconde. Un concept et des pratiques en évolution, M. Verdelhan-Bourgade éd., p.110-122, Bruxelles, De Boeck. 